\title{
Structural physical approximations of unphysical maps and generalized quantum measurements
}

\author{
Jaromír Fiurášek \\ Department of Optics, Palacký University, 17. listopadu 50, 7r200 Olomouc, Czech Republic
}

\begin{abstract}
We investigate properties of the structural physical approximation (SPA) of the partial transposition map recently introduced by Horodecki and Ekert quant-ph/0111064. We focus on the case of two-qubit states and show that in this case the map has the structure of a generalized quantum measurement followed by preparation of a suitable output state. We also introduce SPA for map that transforms two copies of density matrix of a single qubit onto a square of that matrix. We prove that also this map is essentially a generalized quantum measurement.

PACS numbers: 03.65.Bz, 03.67.-a
\end{abstract}

Completely positive $(\mathrm{CP})$ maps represent the most general transformations of quantum states [1]. The fact that quantum dynamics is described by linear CP maps on density matrices can be derived from the static properties of quantum mechanics and the no-signaling condition which states that superluminal communication is excluded [2]. The formalism of $\mathrm{CP}$ maps finds a wide variety of applications in the rapidly growing field of Quantum Information Theory. CP maps are capable to describe an arbitrary quantum transmission channel [3. Construction of devices that optimally approximate some unphysical transformations, such as quantum cloners [4] or universal-NOT gate [5, 6], can be formulated in a unified framework as a determination of an optimal CP map [7]. Properties of probabilistic and deterministic transformations between sets of pure states can be conveniently analyzed with the help of the formalism of $\mathrm{CP}$ maps $[8]$.

The map $\mathcal{E}$ is called positive if for any $\rho \geq 0$ it holds that $\mathcal{E}(\rho) \geq 0$. The map is completely positive iff the induced map $\mathcal{E} \otimes \mathcal{I}_{h}$ is positive for all $h$, where $\mathcal{I}_{h}$ is an identity map on an auxiliary $h$ dimensional Hilbert space. The positive maps that are not completely positive play central role in detecting quantum entanglement. It was shown by Horodeckis [9] that a bipartite state $\rho_{A B}$ is separable if $\left[\mathcal{I}_{A} \otimes \mathcal{M}_{B}\right]\left(\rho_{A B}\right)$ is a positive semidefinite operator for any positive map $\mathcal{M}$ which is not a $\mathrm{CP}$ map. Particularly important is the partial transposition map $\mathcal{P}=\mathcal{I}_{A} \otimes \mathcal{T}_{B}$, whose ability to detect the entanglement was first pointed out by Peres [10]. Since $\mathcal{P}$ is not a CP map, it seems to be impossible to implement this map physically in a lab. Recently, however, an ingenious way how to circumvent this obstacle was suggested by Horodecki and Ekert [11, 12], who introduced the so-called structural physical approximation (SPA) of the unphysical map $\mathcal{P}$. The idea is to form a mixture of the map $\mathcal{P}$ with a $\mathrm{CP}$ map $\mathcal{O}$ that transforms all quantum states onto maximally mixed state, $\mathcal{O}(\rho)=\mathbb{1} / d$, where $\mathbb{1}$ is a unit operator and $\rho$ is an arbitrary operator acting on $d$-dimensional Hilbert space. In the case of the partial transposition map acting on state of two qudits, the optimal SPA is given by 12

$$
\mathcal{P}_{S}=\frac{d^{3}}{d^{3}+1} \mathcal{O}_{A} \otimes \mathcal{O}_{B}+\frac{1}{d^{3}+1} \mathcal{I}_{A} \otimes \mathcal{T}_{B}
$$

Horodecki and Ekert [12] suggested, that the physical trace-preserving $\mathrm{CP}$ map $\mathcal{P}_{S}$ can be utilized to experimentally directly detect the entanglement without the necessity to carry out a full tomographic reconstruction of the bipartite state $\rho_{A B}$ whose entanglement is to be determined. Briefly, the proposed protocol goes as follows. One applies the map (11) to many copies of the bipartite state and then measures the spectrum of the transformed state. This requires determination of no more than $d^{2}-1$ parameters, in contrast to $d^{4}-1$ parameters needed for a full reconstruction of the state $\rho_{A B}$. For example, if one possesses $N$ copies of the state $\rho$, then one can measure the quantity $\mu_{N}=\left\langle\rho^{N}\right\rangle$ which is equal to sum of $N$-th powers of eigenvalues of $\rho$. If the moments $\mu_{N}$, $N=2, \ldots, d^{2}$, are known, then the eigenvalues can be calculated simply by solving a system of algebraic equations. It this context, an interesting question is whether one can find SPA map that would approximate the transformation

$$
\rho^{\otimes N} \rightarrow \rho^{N}
$$

Horodecki conjectured that such SPA trace preserving map does not exist [11].

In this paper we shall analyze the structure of the map $\mathcal{P}_{S}$ acting on two-qubit state, $d=2$. We shall show that $\mathcal{P}_{S}$ can be accomplished by a measurement based scenario: certain generalized measurement is carried out on the two-qubit state $\rho_{A B}$ and the output state is prepared according to the result of this measurement. We shall discuss consequences of this fact for the direct entanglement detection protocol of Horodecki and Ekert. In the second part of the paper we show that SPA of the transformation (2) can be constructed if we allow this map to be trace decreasing. We shall provide an explicit formula for $d=2$ and $N=2$ and it will become clear that an extension to higher dimensions and/or higher powers $N$ is quite straightforward.

Our analysis of the map (1) is based on the well known correspondence between linear completely positive maps and positive semidefinite operators (see, e.g., [7]). Consider a map $\mathcal{E}$ which transforms operators acting on input Hilbert space $\mathcal{H}$ onto operators acting on output Hilbert space $\mathcal{K}$. The map $\mathcal{E}$ is uniquely specified by a positive 
semidefinite operator $E$ defined as follows,

$$
E=[\mathcal{E} \otimes \mathcal{I}](\Phi)
$$

where $\mathcal{I}$ is an identity map, $\Phi=|\phi\rangle\langle\phi|$ and

$$
|\phi\rangle=\sum_{j=1}^{\operatorname{dim} \mathcal{H}}|j\rangle|j\rangle
$$

is a maximally entangled state on $\mathcal{H}^{\otimes 2}$. This correspondence is not only mathematical, the state $E$ can be physically prepared in the lab from the entangled state $\Phi$ by applying the map $\mathcal{E}$ to one part of the state $\Phi$. This allows one, for instance, to transform the problem of tomographic reconstruction of an unknown quantum channel $\mathcal{E}$ onto problem of reconstruction of an unknown quantum state $E[13$, 14], where a variety of well established tomographic techniques may be applied. Moreover, notions established for quantum states can be straightforwardly extended to CP maps. One can define fidelity of two CP maps [15], and consider entanglement [16], storage, compression and purification of the $\mathrm{CP}$ maps [17].

In our case both the input and output Hilbert spaces are spaces of two qubits and the operator $P_{S}$ which represents the map

$$
\mathcal{P}_{S}=\frac{8}{9} \mathcal{O} \otimes \mathcal{O}+\frac{1}{9} \mathcal{I} \otimes \mathcal{T}
$$

can be obtained if we insert (5) into Eq. (3). We show that the map $\mathcal{P}_{S}$ is input-output separable, i.e., that the operator $P_{S}$ can be written as a following convex sum,

$$
P_{S}=\sum_{j} \Pi_{j}^{T} \otimes \rho_{j},
$$

where $T$ stands for the transposition and $\Pi_{j}$ and $\rho_{j}$ are positive semidefinite Hermitian operators on input and output Hilbert spaces $\mathcal{H}$ and $\mathcal{K}$, respectively,

$$
\Pi_{j} \geq 0, \quad \rho_{j} \geq 0 .
$$

Moreover, $\rho_{j}$ represent density matrices, hence their traces are normalized,

$$
\operatorname{Tr}\left[\rho_{j}\right]=1
$$

Furthermore, the operators $\Pi_{j}$ form elements of a positive operator valued measure (POVM) and their sum is a unit operator,

$$
\sum_{j} \Pi_{j}=\mathbb{1}_{\mathcal{H}}
$$

To prove this we recall that the map $\mathcal{P}_{S}$ is trace preserving. In terms of the operator $P_{S}$ this condition reads [7]

$$
\operatorname{Tr}_{\mathcal{K}}\left[P_{S}\right]=\mathbb{1}_{\mathcal{H}}
$$

On inserting the formula (6) into Eq. (10) and making use of the normalization of $\rho_{j}$ we immediately arrive at (9).

Let us now see what are the implications of the separability of the operator $P_{S}$. The relation between input and output density matrices $\rho_{\text {out }}=\mathcal{P}_{S}\left(\rho_{\text {in }}\right)$ can be written with the help of $P_{S}$ as follows $[7$,

$$
\rho_{\text {out }}=\operatorname{Tr}_{\mathcal{H}}\left[P_{S} \rho_{\text {in }}^{T} \otimes \mathbb{1}_{\mathcal{K}}\right]
$$

where $T r_{\mathcal{H}}$ stands for the partial trace over the input Hilbert space. On inserting the convex sum (6) into Eq. (11), we obtain

$$
\rho_{\mathrm{out}}=\sum_{j} \rho_{j} \operatorname{Tr}\left[\rho_{\mathrm{in}} \Pi_{j}\right] .
$$

The interpretation of this expression is rather straightforward, the $\mathrm{CP}$ map $\mathcal{P}_{S}$ is essentially a generalized measurement described by the POVM elements $\Pi_{j}$ and carried out on the input two-qubit state $\rho_{\text {in }}$. If the $j$-th outcome is detected, then the output state is prepared in a state $\rho_{j}$. Notice that $\Pi_{j}$ is detected with the probability $p_{j}=\operatorname{Tr}\left[\rho_{\mathrm{in}} \Pi_{j}\right]$.

We now prove that the operator $P_{S}$ is input-output separable. We do so by explicitly rewriting $P_{S}$ in the form of convex sum of tensor products of positive semidefinite operators in input and output Hilbert spaces. After a somewhat lengthy but straightforward algebra one arrives at the following decomposition,

$$
\begin{aligned}
P_{S}= & \frac{1}{9}\left[W_{0010}^{0010}+W_{0111}^{0111}+W_{0001}^{0100}+W_{0011}^{0110}\right. \\
& +W_{1110}^{1011}+W_{1001}^{1100}+S_{00}^{00}+S_{11}^{11}+S_{10}^{10}+S_{01}^{01} \\
& \left.+2\left(S_{11}^{00}+S_{00}^{11}+S_{10}^{01}+S_{01}^{10}\right)\right],
\end{aligned}
$$

where

$$
W_{i j k l}^{a b c d}=S_{i j}^{a b}+S_{i j}^{c d}+S_{k l}^{a b}+S_{k l}^{c d}+F_{i j k l}^{a b c d}+F_{k l i j}^{c d a b},
$$

and

$$
\begin{gathered}
S_{i j}^{a b}=|i j\rangle\langle i j|\otimes| a b\rangle\langle a b|, \\
F_{i j k l}^{a b c d}=|i j\rangle\langle k l|\otimes| a b\rangle\langle c d| .
\end{gathered}
$$

Here the letters $i, j, k, l$ label states in $\mathcal{H}$ while $a, b, c, d$ denote states in $\mathcal{K}$. We still have to prove that the operators $W_{i j k l}^{a b c d}$ are separable. To do so, we notice that all operators $W$ have the same structure. For the sake of notational simplicity, we make the identification

$$
\begin{array}{rlrl}
|i j\rangle & =|\uparrow\rangle_{1}, & & |k l\rangle=|\downarrow\rangle_{1}, \\
|a b\rangle=|\uparrow\rangle_{2}, & & |c d\rangle=|\downarrow\rangle_{2} .
\end{array}
$$

Thus each operator $W$ represents a state of two qubits. In the basis $|\uparrow \uparrow\rangle_{12},|\uparrow \downarrow\rangle_{12},|\downarrow \uparrow\rangle_{12},|\downarrow \downarrow\rangle_{12}$, the matrix representation of the operator $W$ reads

$$
W=\left(\begin{array}{llll}
1 & 0 & 0 & 1 \\
0 & 1 & 0 & 0 \\
0 & 0 & 1 & 0 \\
1 & 0 & 0 & 1
\end{array}\right)
$$


The separability of the state (16) can be easily proven with the help of partial transposition criterion, because the condition $\left[\mathcal{I}_{1} \otimes \mathcal{T}_{2}\right](W) \geq 0$ is necessary and sufficient for separability of the state $W$. We can even provide an explicit decomposition of $W$ into convex sum of tensor products,

$$
W=\frac{1}{4} \sum_{j=1}^{4}\left|\phi_{j}\right\rangle\left\langle\phi_{j}|\otimes| \psi_{j}\right\rangle\left\langle\psi_{j}\right|,
$$

where the states $\left|\phi_{j}\right\rangle \in \mathcal{H}$ and $\left|\psi_{j}\right\rangle \in \mathcal{K}$ are given by

$$
\begin{aligned}
& \left|\phi_{j}\right\rangle=|\uparrow\rangle+e^{i \frac{\pi}{2} j}|\downarrow\rangle, \\
& \left|\psi_{j}\right\rangle=|\uparrow\rangle+e^{-i \frac{\pi}{2} j}|\downarrow\rangle .
\end{aligned}
$$

This concludes our proof of the input-output separability of the map $\mathcal{P}_{S}$.

We now discuss the implications of our results for the entanglement detection scheme proposed by Horodecki and Ekert. Since the CP map $\mathcal{P}_{S}$ is essentially generalized quantum measurement, the quantum information contained in the two-qubit state $\rho_{\text {in }}$ is converted into a classical information represented by the results of the generalized measurement. Moreover, the preparation of the output state $\rho_{j}$ can be repeated as many times as we wish. The situation here is very similar to the optimal universal-NOT gate, where also the measurement based scenario is the best one [6] and an arbitrary number of the flipped spins can be prepared at the output, all with the same fidelity.

The measurement-based scenario for the CP map $\mathcal{P}_{S}$ also indicates that any subsequent measurement on the output states $\rho_{\text {out }}=\mathcal{P}_{S}\left(\rho_{\text {in }}\right)$ can not reveal any more information in addition to that obtained from the POVM measurement (9). Suppose that we would like to first apply the map (5) to $N$ copies of the two-qubit state $\rho_{\text {in }}$ and then carry out some collective measurement on the output state $\rho_{\text {out }}^{\otimes N}$. We can accomplish this if we perform the measurement (9) on each copy of the input state, then we prepare the output states $\rho_{j}$ according to the measurement results and finally carry out the collective measurement. However, from the results of the measurements (9) on the input states we actually exactly know the particular output state. Hence we need not to prepare the output states $\rho_{j}$ and carry out the collective measurements. Instead, we can simulate the measurement process on a classical computer or even simply calculate mean value of any operator for the particular output state. This implies that the necessity of storage of $N$ copies of the output quantum states is avoided.

Furthermore, the generalized measurement (9) is tomographically complete. This can be easily proved as follows. If we know exactly all the probabilities $p_{j}$ then we can reconstruct the output state $\rho_{\text {out }}$. From this output state we can then obtain the input state $\rho_{\text {in }}$ simply by inverting the linear relation established by the CP map $\mathcal{P}_{S}$. We first subtract a multiple of identity operator and then we apply a partial transposition map (which is its own inverse),

$$
\rho_{\text {in }}=\mathcal{P}\left(9 \rho_{\text {out }}-2 \mathbb{1}\right) .
$$

In this way we recover the input state $\rho_{\text {in }}$. These considerations seem to indicate that a tomographically complete measurement is "hidden" in the CP map $\mathcal{P}_{S}$ used in the scenario for direct detection of the entanglement suggested by Horodecki and Ekert [12]. This tomographically complete measurement also seems to provide more information than any subsequent (possibly collective) measurement on the output states. Nevertheless, the claim of Horodecki and Ekert that their protocol involves determination of a reduced number of parameters in comparison to full tomographic measurement remains valid. However, the noise introduced by the part of the map $\mathcal{P}_{S}$ proportional to $\mathcal{O} \otimes \mathcal{O}$ reduces the amount of information that can be extracted from the (collective) measurements on the output states so it seems that these measurements cannot be more efficient than the POVM (9).

In the rest of the paper, we shall construct an structural physical approximation for the transformation (2). A key observation is that the transformation $\rho^{\otimes N} \rightarrow \rho^{N}$ is in certain sense linear. Indeed, the matrix elements of $\rho^{N}$ are linear combinations of the matrix elements of the operator $\rho^{\otimes N}$. For the sake of simplicity, we shall focus on making square of a density matrix of a single qubit, $d=2$ and $N=2$. Since the map

$$
\mathcal{R}(\rho \otimes \rho)=\rho^{2}
$$

is linear, it can be represented by an operator $R$ according to Eq. (3). We note that the operator $R$ is not positive semidefinite, because $(19)$ is not a CP map. However, we can define the operation $\mathcal{R}$ in such way that it preserves the hermiticity property. This is equivalent to the fact that the corresponding operator $R$ is Hermitian,

$$
\begin{aligned}
R= & {\left[|00\rangle\langle 00|+\frac{1}{2}(|01\rangle\langle 10|+| 10\rangle\langle 01|)\right] \otimes|0\rangle\langle 0| } \\
& +\left[|11\rangle\langle 11|+\frac{1}{2}(|01\rangle\langle 10|+| 10\rangle\langle 01|)\right] \otimes|1\rangle\langle 1| \\
& +\frac{1}{\sqrt{2}}[|00\rangle\langle+|+|+\rangle\langle 11|] \otimes|0\rangle\langle 1| \\
& +\frac{1}{\sqrt{2}}[|+\rangle\langle 00|+| 11\rangle\langle+|] \otimes|1\rangle\langle 0|,
\end{aligned}
$$

where $|+\rangle=(|01\rangle+|10\rangle) / \sqrt{2}$.

Now, it was shown by Horodecki [11, that there exists structural physical approximation for any map that preserves hermiticity property. The explicit construction involves in our case a mixture of the operation $\mathcal{R}$ and an operation $\overline{\mathcal{O}}$ that maps any two-qubit density matrix onto maximally mixed state of a single qubit, $\overline{\mathcal{O}}(\rho)=\mathbb{1} / 2$. The weight of the operation $\overline{\mathcal{O}}$ in the mixture is equal to the lowest eigenvalue of the operator $R$. In this way we 
define SPA operation that optimally approximates the unphysical transformation $\mathcal{R}$,

$$
\mathcal{R}_{S}=\frac{1}{2}(\overline{\mathcal{O}}+\mathcal{R}) .
$$

The operator $R_{S}$ representing CP-map $\mathcal{R}_{S}$ is given by

$$
\begin{aligned}
R_{S}= & \frac{1}{2}\left[\frac{3}{2}|00\rangle\langle 00|+|+\rangle\left\langle+\left|+\frac{1}{2}\right| 11\right\rangle\langle 11|\right] \otimes|0\rangle\langle 0| \\
& +\frac{1}{2}\left[\frac{3}{2}|11\rangle\langle 11|+|+\rangle\left\langle+\left|+\frac{1}{2}\right| 00\right\rangle\langle 00|\right] \otimes|1\rangle\langle 1| \\
& +\frac{1}{2 \sqrt{2}}[|00\rangle\langle+|+|+\rangle\langle 11|] \otimes|0\rangle\langle 1| \\
& +\frac{1}{2 \sqrt{2}}[|+\rangle\langle 00|+| 11\rangle\langle+|] \otimes|1\rangle\langle 0|
\end{aligned}
$$

The CP-map $\mathcal{R}_{S}$ is trace decreasing, which is expressed by the inequality $\operatorname{Tr}_{\mathcal{K}}\left[R_{S}\right]<\mathbb{1}$. Thus the map (21) can be applied only probabilistically (see, e.g., Appendix of Ref. [11]), and the probability of success is given by

$$
P=\frac{1}{2}\left[1+\operatorname{Tr}\left(\rho^{2}\right)\right]
$$

Since the matrix $\rho \otimes \rho$ is symmetric, the input Hilbert space for the transformation $\mathcal{R}_{S}$ is the symmetric subspace of the Hilbert space of two qubits which has dimension 3. The output Hilbert space is, of course, the Hilbert space of a single qubit. We can thus easily check the input-output separability of the CP map $\mathcal{R}_{S}$, because the Peres-Horodecki criterion is necessary and sufficient in this case [9, 10]. We find that the operator $R_{S}$ has positive partial transpose hence is separable. We can write

$$
R_{S}=\sum_{j} \bar{\Pi}_{j}^{T} \otimes \bar{\rho}_{j}
$$

and the map $\mathcal{R}_{S}$ again corresponds to some generalized measurement. However, the map $\mathcal{R}_{S}$ is trace-decreasing, which means that $\sum_{j} \bar{\Pi}_{j}<\mathbb{1}$. The measurement defined by POVM elements $\bar{\Pi}_{j}$ is incomplete and must be completed by adding one more element $\bar{\Pi}_{0}=\mathbb{1}-\sum_{j} \bar{\Pi}_{j}$. The map 21 can be implemented as follows. One carries out the completed measurement $\bar{\Pi}_{j}$ on the input two-qubit state. If $\bar{\Pi}_{0}$ is detected, then the procedure failed. If any other measurement outcome is observed, then the corresponding state $\bar{\rho}_{j}$ is prepared at the output.

In summary, we have studied properties of the structural physical approximation of the partial transposition map on two qubits. We have shown that the SPA map is equivalent to certain generalized quantum measurement. This has consequences for the scenario for direct detection of entanglement proposed by Horodecki and Ekert. In their scheme, collective measurements on several copies of the output states have to be performed. However, these measurements cannot be more efficient than the POVM which is associated with the CP map $\mathcal{P}_{S}$. Our discussion was restricted to the case of two qubits and it would be interesting to see whether the CP map (11) is equivalent to generalized measurement also for higher dimensions. This is an open question which certainly deserves further investigation.

We have also introduced an SPA for the map that makes $\rho^{2}$ from two copies of the density matrix of a single qubit $\rho$. We have shown that this SPA map is trace-decreasing and is also equivalent to some generalized quantum measurement. We expect that the construction of the map $\mathcal{R}_{S}$ can be quite straightforwardly extended to higher dimensions and/or powers, which will be the subject of further investigations.

I would like to thank R. Filip and Z. Hradil for stimulating discussions. This work was supported by Grant No LN00A015 and Research Project CEZ: J14/98 of the Czech Ministry of Education.
[1] K. Kraus, States, Effects, and Operations (SpringerVerlag, Berlin, 1983).

[2] C. Simon, V. Bužek, and N. Gisin, Phys. Rev. Lett. 87, 170405 (2001).

[3] B. Schumacher, Phys. Rev. A 54, 2614 (1996).

[4] V. Bužek and M. Hillery, Phys. Rev. A 54, 1844 (1996); N. Gisin and S. Massar, Phys. Rev. Lett. 79, 2153 (1997); D. Bruss, A. Ekert, and C. Macchiavello, Phys. Rev. Lett. 81, 2598 (1998); R.F. Werner, Phys. Rev. A 58, 1827 (1998).

[5] N. Gisin and S. Popescu, Phys. Rev. Lett. 83, 432 (1999).

[6] V. Bužek, M. Hillery, and R.F. Werner, Phys. Rev. A 60, 2626 (1999); J. Mod. Opt. 47, 211 (2000).

[7] J. Fiurášek, Phys. Rev. A 64, 062310 (2001).
[8] A. Chefles, arXiv:quant-ph/0109060.

[9] M. Horodecki, P. Horodecki, and R. Horodecki, Phys. Lett. A223, 1 (1996).

[10] A. Peres, Phys. Rev. Lett. 77, 1413 (1996).

[11] P. Horodecki, arXiv: quant-ph/0111036.

[12] P. Horodecki and A. Ekert, arXiv: quant-ph/0111064.

[13] D.G. Fischer, M. Mack, M.A. Cirone, and M. Freyberger, Phys. Rev. A 64, 022309 (2001).

[14] M.G.A. D'Ariano and P. Lo Presti, Phys. Rev. Lett. 86, 4195 (2001).

[15] M. Raginsky, Phys. Lett. A290, 11 (2001).

[16] P. Zanardi, Phys. Rev. A 63, 040304(R) (2001).

[17] W. Dür and J. I. Cirac, Phys. Rev. A 64, 012317 (2001). 